DOI: 10.12957/demetra.2015.13918

\title{
Adolescentes e alimentos diet e light: definição, frequência e razões para o consumo
}

Adolescents and diet and light food: definition, frequency and reasons for food consumption

Daiane Pires da Silva Santos

Maria Luisa de Jesus Barbosa'

Janine Vitorasse Delbonil

Márcia Lopes Weber ${ }^{r}$

${ }^{1}$ Centro Universitário Adventista de São PauloUNASP, Curso de Nutrição. São Paulo-SP, Brasil

Correspondência / Correspondence Márcia Lopes Weber

E-mail: marciaws@yahoo.com.br

\section{Resumo}

O objetivo do estudo foi caracterizar o consumo de alimentos diet e light em adolescentes de escolas pública e privada, identificando o grau de conhecimento acerca do conceito destes alimentos, frequência e razóes para o consumo. Foi realizado estudo transversal com 284 adolescentes entre 13 a 18 anos de ambos os sexos, matriculados em uma escola pública e uma escola privada na zona sul de São Paulo/SP. Foi aplicado questionário de auto-resposta contendo questões objetivas sobre identificação dos conceitos de alimentos diet e light, frequência e motivos para consumi-los. As respostas de ambos os sexos para consumo de alimentos diet e light foram similares entre si no mesmo tipo de escola. O consumo de alimentos diet e light foi maior entre os adolescentes da escola privada em relação aos da pública, em ambos os sexos. Alimentos light eram consumidos em maior proporção que diet em ambos os sexos e escolas e na escola pública meninos consumiam alimentos diet e light em maior proporção que as meninas, o que inverteu-se na escola privada. Em ambas as escolas, a frequência de consumo semanal foi maior entre os meninos. Embora a maioria dos adolescentes de ambas as escolas e sexos tenham optado pelo conceito correto para alimentos diet e light, eles demonstraram não saber diferenciar esses alimentos. O motivo mais citado para o consumo destes alimentos por meninos de ambas as escolas e meninas da escola privada foi benefício à saúde, e para as meninas da escola pública foi manutenção do peso.

Palavras-chave: Consumo de Alimentos. Comportamento de Adolescente. Adoçantes Dietéticos. 


\section{Abstract}

The aim of this study was to characterize the diet and light food consumption in adolescents from public and private schools, and to identify the level of knowledge about these foods definition, frequency and reasons for its consumption. A cross-sectional study was conducted with 284 adolescents aged 13-18 years, boys and girls, in a public school and a private school in the south of São Paulo/SP. The self-report questionnaire was applied with objective questions about the definition of diet and light foods, frequency and reasons to its consumption. The answers of boys and girls about diet and light food consumption were similar for each type of school. The diet and light food consumption was higher among adolescents from private school than in public school, both boys and girls. The light food was consumed in greater proportion than food diet in both sexes and schools. Public school boys consumed diet and light food in greater proportion than girls, which was the oppositte in the private school. In both schools, the frequency of weekly consumption was higher among boys. Although most adolescents of both sexes and schools have opted for the correct concept for diet and light food, they demonstrated not knowing how to identify differences in these foods. The most mentioned reason for the diet and light food consumption by adolescents from both schools and private school girls was health benefits, and for public school girls was weight maintenance.

Key words: Food Consumption. Adolescent Behavior. Dietetic Sweeteners.

\section{Introdução}

O consumo de alimentos diet e light cresceu significativamente nos últimos anos e vem aumentando seu espaço na mesa do consumidor. ${ }^{1-3} \mathrm{~A}$ procura por estes alimentos, além de abranger consumidores especiais, inclui também pessoas interessadas em manter a saúde e a boa forma física. ${ }^{4}$

De acordo com a legislação, alimentos diet são aqueles que não contam com possuem ausência de um determinado ingrediente, como sacarose, proteína, gordura ou sódio, de maneira que sua composição atenda as necessidades de pessoas em condições metabólicas, fisiológicas ou patológicas

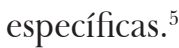

Para ser considerado light, um alimento deve apresentar redução mínima de 25\% de um determinado ingrediente ou nutriente quando comparado ao alimento convencional, podendo 
envolver valor energético, açúcar, sódio, gorduras totais ou saturadas, e colesterol. ${ }^{6} \mathrm{O}$ termo light constitui-se em um tipo de informação nutricional complementar para alimentos industrializados, é sinônimo de reduzido e deve estar seguido, no rótulo do alimento, da informação de quanto foi a redução e sobre qual nutriente esta informação refere-se. ${ }^{6}$

A população tem demonstrado falta de conhecimento com relação à utilização destes produtos. Entre indivíduos adultos, a procura está relacionada à manutenção de uma boa saúde e os mais jovens buscam estes produtos por preocuparem-se com a aparência corporal. ${ }^{7} \mathrm{Na}$ adolescência, essa preocupação parece ser maior devido às mudanças que ocorrem no corpo do adolescente, decorrentes do processo de crescimento e desenvolvimento. ${ }^{8}$

A adolescência consiste em um período de transição da infância para a vida adulta, compreende a idade entre 10 e 19 anos, e é marcado por mudanças biológicas, psicológicas, cognitivas e sociais que interferem no comportamento alimentar do adolescente. ${ }^{9,10}$ São vários os fatores que interferem no consumo alimentar na adolescência, como valores socioculturais, imagem corporal, convivências sociais, situação financeira, consumo de alimentos fora de casa, aumento do consumo de alimentos semipreparados, influência da mídia, hábitos alimentares, disponibilidade de alimentos, e facilidade de preparo. ${ }^{11}$

Devido à ampla distribuição de produtos diet e light no mercado, juntamente com a preocupação ligada à estética, os adolescentes têm apresentado consumo crescente destes produtos. ${ }^{12}$ Há relatos de que os adolescentes utilizam estes produtos com o intuito de perda ou manutenção de peso corporal, por vezes sem o conhecimento adequado para esta escolha. ${ }^{13}$

O objetivo deste estudo foi caracterizar o consumo de alimentos diet e light em adolescentes de escolas pública e privada, identificando o grau de conhecimento acerca do conceito destes alimentos, frequência e razões para o consumo.

\section{Metodologia}

Trata-se de um estudo transversal, realizado em amostra de conveniência. Participaram do estudo adolescentes com faixa etária entre 13 e 18 anos, de ambos os sexos, matriculados em uma escola pública e uma escola privada, ambas localizadas no mesmo bairro da zona sul de São Paulo/SP. Todos os adolescentes da faixa etária em estudo, em ambas as escolas, foram convidados a participar. O estudo foi aprovado pelo Comitê de Ética em Pesquisa do Centro Universitário Adventista de São Paulo, sob protocolo 20869913.9.0000.5377.

A amostra foi composta de 210 adolescentes na escola pública (21\% da população, sendo 99 meninas) e 74 na escola privada (30\% da população, sendo 37 meninas). As perdas deveram-se à 
recusa dos adolescentes em participar, à entrega do Termo de Consentimento Livre e Esclarecido sem a assinatura dos pais ou responsáveis, à ausência nos dias de coleta de dados e ao preenchimento incompleto do instrumento utilizado. Como critério de exclusão, foi determinado que seriam excluídos do estudo adolescentes portadores de Diabetes mellitus, o que não ocorreu na amostra. A coleta de dados ocorreu entre outubro e dezembro de 2013.

Foi aplicado questionário desenvolvido por Santos, ${ }^{7}$ devidamente adaptado aos objetivos deste estudo. O instrumento era de auto-resposta e continha questões objetivas referentes à frequência e motivo para eventual consumo, além de noção dos adolescentes acerca dos conceitos de alimentos diet e light. O questionário foi aplicado em sala de aula, em horário determinado pela direção das escolas, com apoio e acompanhamento dos pesquisadores.

Para análise dos dados, foram comparadas as respostas de ambos os sexos por tipo de escola e de ambos os sexos, entre si e na mesma escola. Para comparação dos resultados entre os sexos e entre os tipos de escolas, foi aplicado o teste do Qui-quadrado de Pearson, adotando-se p<0,05 como nível de significância.

\section{Resultados}

A caracterização do consumo e identificação dos conceitos de alimentos diet e light pelos adolescentes, comparando-se os sexos por tipo de escola, estão apresentados na Tabela 1. A proporção de meninas que não consumia alimentos diet foi significantemente maior na escola pública que na escola privada ( $\mathrm{p}=0,0305)$, com 43,2\% destas últimas relatando consumo. A maioria das meninas que relatou consumir este tipo de alimento mencionou frequência de consumo de 1 a 3 vezes na semana na escola privada $(54,1 \%)$ e raramente, na escola pública $(43,8 \%)$, sem diferença estatisticamente significante em nenhuma frequência. A proporção de meninas que escolheu o conceito correto de alimento diet foi similar em ambas as escolas (62,2\% na escola privada e 55,6\% na pública), sem diferença significante. 
Tabela 1. Caracterização do consumo e identificação dos conceitos de alimentos diet e light pelos adolescentes, segundo sexo e tipo de escola. São Paulo-SP, 2013/2014.

\begin{tabular}{ccccccc}
\hline & \multicolumn{3}{c}{ Sexo Feminino } & \multicolumn{3}{c}{ Sexo Masculino } \\
\cline { 2 - 6 } & Pública & Privada & Pública & Privada & \\
$(\mathrm{n}=99)$ & $(\mathrm{n}=37)$ & $p^{*}$ & $(\mathrm{n}=111)$ & $(\mathrm{n}=37)$ & $p^{*}$ \\
$\mathrm{n}(\%)$ & $\mathrm{n}(\%)$ & & $\mathrm{n}(\%)$ & $\mathrm{n}(\%)$ & \\
\hline
\end{tabular}

Alimentos Diet

\section{Consome}

Sim

Não

Frequência de consumo

1 a 3 vezes/semana

4 a 7 vezes/semana

Raramente

Conceito

Correto

Incorreto

$$
\begin{array}{ll}
24(24,2) & 16(43,2) \\
75(75,8) & 21(56,8)
\end{array}
$$

$\begin{array}{llll}0,0305 & 34(30,6) & 12(32,4) & \\ & 77(69,4) & 25(67,6) & 0,8371\end{array}$

\section{Alimentos Light}

\section{Consome}

Sim

Não

$\begin{array}{rrrrrr}13(54,1) & 5(31,2) & 0,1535 & 16(47,0) & 5(41,7) & 0,7472 \\ 2(8,4) & 4(25,0) & 0,1481 & 6(17,8) & 2(16,6) & 0,9588 \\ 9(37,5) & 7(43,8) & 0,6926 & 12(35,2) & 5(41,7) & 0,6942\end{array}$

$55(55,6) \quad 24(62,2)$

$44(44,4) \quad 13(37,8)$

$59(53,2) \quad 23(62,2)$

$\begin{array}{lllll}0,3275 & 0,3397\end{array}$ $52(46,8) \quad 14(37,8)$

\section{Frequência de consumo}

$\begin{array}{lrlllll}1 \text { a } 3 \text { vezes/semana } & 15(36,5) & 8(32,0) & 0,7045 & 17(33,3) & 9(39,1) & 0,6288 \\ 4 \text { a } 7 \text { vezes/semana } & 6(14,7) & 8(32,0) & 0,0941 & 11(21,6) & 12(52,2) & 0,0085 \\ \text { Raramente } & 20(48,8) & 9(36,0) & 0,3102 & 23(45,1) & 2(8,7) & 0,0022\end{array}$

\section{Conceito}

Correto

$$
51(51,5) \quad 21(56,7)
$$

Incorreto

$$
\begin{array}{llllll}
41(41,4) & 25(67,5) & 0,0066 & 51(46,0) & 23(62,1) & \\
58(58,6) & 12(32,5) & & 60(54,0) & 14(37,9) & 0,0875
\end{array}
$$

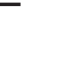




\begin{tabular}{lcccccc}
\hline & \multicolumn{3}{c}{ Sexo Feminino } & \multicolumn{3}{c}{ Sexo Masculino } \\
\cline { 2 - 6 } & $\begin{array}{c}\text { Pública } \\
(\mathrm{n}=99)\end{array}$ & $\begin{array}{c}\text { Privada } \\
(\mathrm{n}=37)\end{array}$ & $p^{*}$ & $\begin{array}{c}\text { Pública } \\
(\mathrm{n}=111)\end{array}$ & $\begin{array}{c}\text { Privada } \\
(\mathrm{n}=37)\end{array}$ & $p^{*}$ \\
& $\mathrm{n}(\%)$ & $\mathrm{n}(\%)$ & & $\mathrm{n}(\%)$ & $\mathrm{n}(\%)$ & \\
\hline $\begin{array}{l}\text { Motivo do consumo/ } \\
\quad \text { diet e light }\end{array}$ & & & & & & \\
Faz bem à saúde \\
$\begin{array}{l}\text { Prescrição médica ou } \\
\text { nutricionista }\end{array}$
\end{tabular}

*Teste do Qui-quadrado

Em relação aos alimentos light, a proporção de meninas da escola privada que relatou consumo foi significantemente maior que na escola pública (67,5\% e 41,4\%, respectivamente; com p=0,0066). Entre as meninas que consumiam estes alimentos, a frequência de consumo com maior proporção foi raramente, em ambas as escolas e sem diferença significante (36,6\% na escola privada e 48,8\% na pública). O conceito correto de alimento light foi assinalado em proporção similar pelas meninas de ambas as escolas, e envolveu cerca de metade das participantes $(56,7 \%$ na escola privada e $51,5 \%$ na pública). Quando questionadas sobre as diferenças entre alimentos diet e light, a maioria das meninas em ambas as escolas disseram tratar-se do mesmo produto (73/99 na escola pública e 24/37 na escola privada; dados não mostrados).

A maioria das meninas da escola privada (69,7\%) assinalou benefícios dos alimentos diet e light à saúde como motivo para o consumo destes produtos em proporção significantemente maior que a observada nas respostas das meninas da escola pública $(p=0,0106)$. Estas últimas mencionaram motivo relacionado à contribuição destes alimentos na manutenção do peso em maior proporção $(56,8 \%)$, resultado estatisticamente significante na comparação com as meninas da escola privada $(\mathrm{p}=0,0033)$.

Entre os meninos, a maioria em ambas as escolas relatou não consumir alimentos diet (67,6\% na escola privada e 69,4\% na pública), sem diferença significante. A frequência de consumo de 1 a 3 vezes na semana foi assinalada em maior proporção pelos meninos de ambas as escolas (41,7\% na escola privada e $47 \%$ na pública), sem diferença significante. Em relação ao conceito de alimento diet, a maioria dos meninos de ambas as escolas escolheu o conceito correto $(62,2 \%$ na escola privada e 53,2\% na pública), também sem diferença significante. 
A proporção de meninos que não consumia alimentos light foi maior na escola pública que na escola privada $(p=0,0875)$. Entre os meninos que relataram consumir este tipo de alimento, as diferenças entre o tipo de escola surgiu nas frequências de consumo 4 a 7 vezes na semana (maior proporção na escola privada, com p=0,0085) e raramente (maior proporção na escola pública, com $\mathrm{p}=0,0022)$. À semelhança do observado entre as meninas, a maioria dos meninos de ambas as escolas assinalou o conceito correto para alimento light (64,8\% na escola privada e $55 \%$ na pública). Proporções similares foram observadas entre meninos de ambas as escolas também na questão referente às diferenças entre alimentos diet e light, em que a maioria mencionou tratar-se de produtos similares (92/111 na escola pública e 26/37 na escola privada; dados não mostrados).

Como motivo para o consumo dos alimentos diet e light, a maioria dos meninos de ambas as escolas mencionou benefícios destes produtos à saúde (73\% na escola privada e 56,7\% na pública), sem diferença significante.

A Tabela 2 apresenta a caracterização do consumo de alimentos diet e light e a identificação do conceito destes alimentos entre os adolescentes, comparando-se as respostas dos adolescentes de ambos os sexos no mesmo tipo de escola. As proporções de respostas relacionadas a todas as questões foram similares entre os sexos, dentro do mesmo tipo de escola, sem diferença estatisticamente significante na maiorida das questões. As diferenças surgiram na frequência de consumo semanal de alimentos light, na escola privada, em que as meninas relataram a frequência raramente em proporção significantemente maior que os meninos $(p=0,0245)$. Observou-se diferença estatisticamene significante na proporção de meninas em relação aos meninos, na escola pública, que mencionaram como motivo para o consumo de alimentos diet e light sua contribuição na manutenção do peso $(\mathrm{p}=0,0228)$.

Tabela 2. Caracterização do consumo e identificação dos conceitos de alimentos diet e light pelos adolescentes, segundo tipo de escola e sexo. São Paulo-SP, 2013/2014.

\begin{tabular}{|c|c|c|c|c|c|}
\hline \multicolumn{2}{|c|}{ Escola Pública } & \multicolumn{4}{|c|}{ Escola Privada } \\
\hline $\begin{array}{l}\text { Feminino } \\
\qquad(\mathrm{n}=99)\end{array}$ & $\begin{array}{c}\text { Masculino } \\
(\mathrm{n}=111)\end{array}$ & $p^{*}$ & $\begin{array}{c}\text { Feminino } \\
\qquad(\mathrm{n}=37)\end{array}$ & $\begin{array}{l}\text { Masculino } \\
\qquad(\mathrm{n}=37)\end{array}$ & $p^{*}$ \\
\hline n $(\%)$ & n $(\%)$ & & n $(\%)$ & n $(\%)$ & \\
\hline
\end{tabular}

Alimentos Diet

\section{Consome}

Sim

$24(24,2) \quad 34(30,6)$

Não

$75(75,8) \quad 77(69,4)$

0,3013

$16(43,2) \quad 12(32,4)$

$21(56,8) \quad 25(67,6)$

0,3377 


\begin{tabular}{ccccccc}
\hline & \multicolumn{2}{c}{ Escola Pública } & \multicolumn{3}{c}{ Escola Privada } \\
\cline { 2 - 6 } & $\begin{array}{ccccc}\text { Feminino } \\
(\mathrm{n}=99)\end{array}$ & $\begin{array}{c}\text { Feminino } \\
(\mathrm{n}=111)\end{array}$ & $p^{*}$ & $(\mathrm{n}=37)$ & $(\mathrm{n}=37)$ & $p^{*}$ \\
$\mathrm{n}(\%)$ & $\mathrm{n}(\%)$ & & $\mathrm{n}(\%)$ & $\mathrm{n}(\%)$ & \\
\hline
\end{tabular}

\section{Frequência de consumo}

1 a 3 vezes/semana

$5(31,2)$

$5(41,7)$

0,5692

4 a 7 vezes/semana

$2(8,4)$

$6(17,6)$

$0,3110 \quad 4(25,0)$

$2(16,6)$

0,5949

Raramente

$9(37,5)$

$12(35,3)$

$0,8647 \quad 7(43,8)$

$5(41,7)$

0,9195

\section{Conceito}

Correto

$55(55,6) \quad 59(53,2)$

Incorreto

$44(44,4) \quad 52(46,8)$

$24(64,9) \quad 23(62,2)$

0,7272

$13(35,1) \quad 14(37,8)$

0,8094

Alimentos Light

\section{Consome}

Sim

$$
\begin{array}{llllll}
41(41,4) & 51(46,0) & & 25(67,5) & 23(62,1) & \\
58(58,6) & 60(54,0) & 0,5088 & 12(32,5) & 14(37,9) & 0,6263
\end{array}
$$

\section{Frequência de consumo}

$\begin{array}{lrrrrrr}1 \text { a } 3 \text { vezes/semana } & 15(36,5) & 17(33,3) & 0,7045 & 8(32,0) & 9(39,1) & 0,6288 \\ 4 \text { a } 7 \text { vezes/semana } & 6(14,7) & 11(21,6) & 0,3943 & 8(32,0) & 12(52,2) & 0,1567 \\ \text { Raramente } & 20(48,8) & 23(45,1) & 0,7250 & 9(36,0) & 2(8,7) & 0,0245\end{array}$

\section{Conceito}

Correto

$51(51,5) \quad 61(55,0)$

0,6179

$21(56,7) \quad 24(64,8)$

$16(43,3) \quad 13(35,2) \quad 0,4750$

Incorreto

$48(48,5) \quad 50(45,0)$

Motivo do

consumo/diet e light

Faz bem à saúde

$21(41,2) \quad 38(56,7) \quad 0,0944 \quad 23(69,7) \quad 19(73,0) \quad 0,7761$

Prescrição médica ou nutricionista

$$
1(2,0) \quad 5(7,5) \quad 0,3227 \quad 2(6,1) \quad 2(7,7) \quad 0,8047
$$

Não engorda

$29(56,8) \quad 24(35,8) \quad 0,0228 \quad 8(24,2) \quad 5(19,3) \quad 0,6447$

*Teste do Qui-quadrado 


\section{Discussão}

Neste estudo, as respostas de ambos os sexos para consumo de alimentos diet e light foram similares entre si dentro do mesmo tipo de escola, tanto na escola privada quanto na pública. Houve diferenças, no entanto, quando foram comparadas as respostas do mesmo sexo por tipo de escola. Isto sugere um comportamento semelhante em meninos e meninas dentro da escola de origem, mas diferente em algumas questões quando comparados por tipo de escola.

O consumo de alimentos diet e light foi maior entre os adolescentes da escola privada em relação aos da pública, em ambos os sexos. Estudo desenvolvido por Santos ${ }^{7}$, com 155 adolescentes de escolas privadas e públicas, também observou maior consumo de alimentos diet e light na escola privada. No presente estudo, alimentos light eram consumidos em maior proporção que alimentos diet em ambos os sexos e escolas. Na escola pública, meninos consumiam tanto alimentos diet quanto light em maior proporção que as meninas, o que inverteu-se na escola privada. O maior consumo pelos meninos na escola pública difere dos achados de Novaes, ${ }^{14}$ que observou maior consumo de alimentos diet e light entre adolescentes do sexo feminino também em escola pública. Já os resultados da escola privada alinharam-se com o observado por Meira, ${ }^{15}$ em que adolescentes do sexo feminino relataram consumo dos dois tipos de alimentos em maior proporção que os meninos.

Pesquisa desenvolvida em Cotia/SP concluiu que adolescentes de ambos os sexos consumiam refrigerantes em duas ou 3 refeições diárias, tanto na versão adoçada com açúcar quanto aqueles contendo adoçantes artificiais. ${ }^{16}$ A Pesquisa de Orçamentos Familiares 2008-2009, ${ }^{17}$ considerando todas as regiões brasileiras, identificou consumo total de refrigerantes diet e light por 40,1\% dos participantes, e de pães e similares, também nas versões diet e light, de 48,3\%. Entre 2000 e 2010, observou-se declínio nas compras de alimentos adoçados com açúcar nos Estados Unidos, paralelamente ao crescimento de alimentos contendo edulcorantes artificiais, e isto foi mais significativo em famílias que tinham crianças em casa. ${ }^{18}$ Estudo realizado em Caxias do Sul/RS com adultos de ambos os gêneros observou consumo de alimentos diet por $26 \%$ dos participantes, e light, de $41 \% .{ }^{19}$ Estas proporções foram similares aos relatos de consumo dos adolescentes da escola pública do presente estudo (27\% e 44\%, respectivamente) e menores que os da escola privada (38\% e 65\%, respectivamente). Todos estes dados demonstram que a aquisição de alimentos diet e light pela população é considerável, e que este comportamento repete-se em diferentes faixas etárias e regiões brasileiras.

A presença significativa de mulheres no mercado de consumo de produtos diet e light parece não estar restrita a adolescentes ou ao Brasil, como observado no presente estudo e nas demais pesquisas mencionadas. Estudo americano, que analisou a aquisição de alimentos e bebidas 
contendo adoçantes artificiais nos Estados Unidos, entre 2007 e 2008, constatou que a proporção de consumo destes produtos aumentou significativamente no sexo feminino, incluindo mulheres adolescentes e adultas, e no sexo masculino manteve-se estável. ${ }^{20}$

Raramente foi a frequência de consumo mencionada em maior proporção pelas meninas da escola privada tanto para alimentos diet quanto para light. Santos ${ }^{7}$ observou essa mesma frequência na escola privada. No entanto, ao somar os intervalos de frequência de consumo de 1 a 3 e de 4 a 7 vezes na semana, a proporção observada no presente estudo alcançou mais que $50 \%$ dos adolescentes, em ambos os sexos e escolas, tanto para alimentos diet quanto para light. Considerando ainda frequência de consumo de 1 a 7 vezes na semana, os meninos apresentaram maior proporção que as meninas em ambas as escolas, chegando a 91,3\% na escola privada. No estudo de $\mathrm{Santos}^{7}$, os adolescentes também apresentaram proporção acima de $50 \%$ para frequência semanal de consumo de 1 a 7 vezes, e frequências similares em ambos os tipos de escola.

Embora a maioria dos adolescentes de ambas as escolas e sexos tenham optado pelo conceito correto tanto para alimentos diet quanto para light, eles demonstraram não saber diferenciar esses alimentos entre si. Proporção menor foi observada em estudo de Branco, ${ }^{12}$ em que 38\% e 33\% dos adolescentes identificaram corretamente os conceitos de alimentos diet e light, respectivamente. Os resultados do presente estudo em relação à identificação do conceito também foram mais significativos que os de pesquisa desenvolvida por Tebaldi, ${ }^{13}$ envolvendo adolescentes de escola pública em Corbélia-PR, e observou que o conceito correto de alimento diet foi mencionado por 12\% de meninas e 51,3\% de meninos, e em relação a light, responderam corretamente $28 \%$ das meninas e 48,9\% dos meninos. Em relação à diferenciação de diet e light, resultados de outras pesquisas confirmam o observado no presente estudo, em proporções similares. Meira ${ }^{15}$ identificou que adolescentes de ambos os sexos desconheciam a diferença entre os dois tipos de produtos. Entre adultos, Nunes ${ }^{19}$ também verificou dúvidas na diferenciação destes alimentos, além de proporções baixas de conhecimento sobre o conceito de light (39\%) e diet (44\%).

Segundo Branco, ${ }^{21}$ a população apresenta dificuldade de discernimento em relação à utilização adequada de produtos diet e light, o que também foi identificado entre os adolescentes do presente estudo. Tanto o acesso quanto a compreensão sobre o conteúdo e o tipo de alimento industrializado, assim como sua indicação de uso, configuram questão de segurança alimentar e nutricional, além de contribuir com a saúde da população. Se as diferenças entre alimentos diet e light não estiverem claras, o consumo destes produtos pode causar danos à saúde dos indivíduos, como o uso por diabéticos de açúcares denominados light e que contêm sacarose em sua fórmula. Apenas a menção aos termos diet e light nos rótulos dos produtos é insuficiente para que o consumidor identifique suas diferenças e indicações de consumo. ${ }^{19}$ 
As meninas da escola pública revelaram optar por alimentos diet e light pela sua contribuição na manutenção do peso corporal, e as meninas da escola privada, por seus benefícios à saúde. A maioria dos meninos de ambas as escolas mencionou benefícios à saúde como razão para consumir estes produtos. Estas duas razões para consumo foram relatadas em várias pesquisas similares. Santos ${ }^{7}$ identificou que a maioria dos adolescentes, de escolas privadas e públicas, relatou motivação relacionada ao fato de acreditarem que estes alimentos fazem bem à saúde, seguido pela sua contribuição na manutenção ou perda de peso. Da mesma forma, Meira ${ }^{15}$ observou, com adolescentes de escola privada de João Pessoa/PB, que as meninas escolhiam esses produtos para emagrecer, e os meninos por considerarem que são saudáveis. Outros estudos similares demonstraram que as maiores motivações para a aquisição de produtos diet e light são a preocupação com a saúde, ${ }^{22}$ além de particularidades ligadas a enfermidades específicas para alimentos diet, e busca de alimentação saudável para alimentos light. ${ }^{19}$ De acordo com Novaes,${ }^{14}$ as meninas tendem a preocupar-se mais que os meninos com o peso corporal, o que foi também observado no presente estudo, em que 27,2\% das meninas de ambas as escolas relataram escolher estes produtos em razão de manutenção de peso, e 19,5\% dos meninos mencionaram a mesma razão. Isto reflete-se na escolha de alimentos, permitindo identificar por vezes o uso indiscriminado e inadequado de produtos diet e light pela população jovem, com intuito de perda e/ou manutenção do peso corporal. ${ }^{12}$ A adolescência é um período em que ocorrem grandes modificações corporais, acrescidas de preocupações dos jovens com sua imagem corporal e com a busca de um corpo ideal e adequado aos padrões sociais. A relação entre a satisfação com a imagem corporal e o consumo de alimentos diet e light já foi identificada em adolescentes, observando-se que independente do sexo, quanto maior o grau de insatisfaçãao corporal, maior é o consumo destes produtos. ${ }^{21}$

O papel dos alimentos contendo adoçantes artificiais na redução do peso corporal é controverso. O consumo de bebidas industrializadas com elevado teor de açúcar apresentou associação com o excesso de peso em estudos envolvendo crianças e adolescentes. ${ }^{23,24}$ Adicionalmente, há pesquisas que observaram que consumo de bebidas contendo adoçantes artificiais por adolescentes também apresenta associação com excesso de peso $^{25,26}$ e com dieta elevada em calorias, carboidratos, gorduras e alimentos ricos em sódio e açúcar. ${ }^{27}$ Os mecanismos fisiológicos relacionados ao uso de adoçantes artificiais e saciedade ou redução de peso corporal não estão totalmente esclarecidos, e nem há consenso sobre eles. Evidências sugerem que a capacidade do organismo para compensar calorias ingeridas não é eficiente e/ou constante, independente do alimento ser sólido ou líquido, e que o indivíduo pode buscar compensar as calorias reduzidas de alimentos contendo adoçantes artificiais na próxima refeição, tornando-a mais calórica. ${ }^{28}$ Isto explicaria em parte as evidências de que alimentos contendo adoçantes artificiais apresentam fraca associação com perda e manutenção do novo peso corporal, e também associam-se a ganho de peso. ${ }^{28-30}$ 


\section{Conclusão}

Observou-se por meio deste estudo que os adolescentes da escola privada apresentaram maior consumo de alimentos diet e light em relação aos da escola pública, independente do sexo. Comparando os sexos no mesmo tipo de escola, verificou-se maior proporção de consumo de alimentos diet e light dos meninos em relação às meninas na escola pública, o que inverteu-se na escola privada. Em ambas as escolas, a frequência de consumo semanal foi maior entre os meninos. Apesar da maioria dos adolescentes identificar corretamente os conceitos tanto de diet quanto de light, foi pequena a proporção dos que mencionaram haver diferença entre estes tipos de alimentos. O motivo mais citado para o consumo destes alimentos por meninos de ambas as escolas e meninas da escola privada foi benefício à saúde, e para as meninas da escola pública foi manutenção do peso.

Sugere-se o desenvolvimento e a adoção de intervenções educativas nas escolas, com foco na orientação e esclarecimento dos adolescentes acerca da finalidade e consumo adequado e consciente destes alimentos.

\section{Referências}

1. Faria MG, Caldeira FVND, Assis EM, Rodrigues FC, Fujii JB. Avaliação do nível de conhecimento dos consumidores de Ipatinga/MG, sobre as características e propriedades de produtos diet e light e a diferença entre ambos. Hig. Aliment. 2007; 21(153):34-38.

2. Gonçalves JA, Zucchi ND, Abreu TC, Caldart S, Batista SMM, Fiates GMR. Alimentos diet e light: consumo e conhecimento por frequentadores de supermercados de Florianópolis/SC. Hig. Aliment. 2013; 27(216):124-128.

3. Lucchese T, Batalha MO, Lambert JL. Marketing de alimentos e o comportamento de consumo: proposição de uma tipologia do consumo de produtos light e diet. Organizações Rurais \& Agroindustriais 2009; 8(2):227-239.

4. Sousa EP. Comportamento do consumidor de produtos diet e light na cidade de Fortaleza/CE. In: Anais, XLIII Congresso Brasileiro de Economia e Sociologia Rural; 2005; Ribeirão Preto. Brasília: SOBER; 2005. p. 1-11.

5. Brasil. Portaria no 29, de 13 de janeiro de 1998. Regulamento técnico para fixação de identidade e qualidade de alimentos para fins especiais. Diário Oficial da União, 13 jan. 1998.

6. Brasil. Agência Nacional de Vigilância Sanitária. Resolução RDC no 54, de 12 de novembro de 2012. Regulamento técnico sobre informação nutricional compementar. Diário Oficial da União, 12 nov. 2012.

7. Santos VS, Miquelanti VP. Estado nutricional e consumo de alimentos diet e light em adolescentes de escolas públicas e privadas de Patos de Minas/MG. Rev. Min. Ciênc. Saúde 2009; 1(1):101-120. 
8. Almeida CF, Pereira RBC, Bittencout A, Ribeiro RL, Coelho SC. Frequência de consumo alimentar versus saúde de adolescentes. Rede Cuid. Saúde 2009; 3(3):1-12.

9. Vitolo MR. Nutrição: da gestação à adolescência. Rio de Janeiro: Rubio; 2008.

10. Abreu WC, Coelho TM, Souza RA, Guimarães LC. Nutritional status, knowledge about nutrition and feeding practices of adolescents enrolled in high schools of the town of Formiga/MG. Alim. Nutr. 2012; 23(4):521-527.

11. Garcia GCB, Gambardella AMD, Frutuoso MFP. Estado nutricional e consumo alimentar de adolescentes de um centro de juventude da cidade de São Paulo. Rev. Nutr. 2003; 16(1):41-50.

12. Branco LM, Passos MAZ, Almeida EC. Conhecimento e consumo de alimentos diet e light por adolescentes. Nutr. Pauta 2005; (73):20-24.

13. Tebaldi P, Carvalho HSL. Conhecimento e consumo de diet e light por adolescentes matriculados em um colégio estadual de Corbélia/PR [trabalho de conclusão de curso]. Cascavel: Faculdade Assis Gurgacz, Curso de Nutrição; 2006.

14. Novaes JF, Franceshini SCC, Priore SE. Utilização de dietas e consumo de alimentos diet e light entre adolescentes de escola privada do município de Viçosa-MG. Nutrire 2004; (28):25-35.

15. Meira CC, Tertuliano AF, Rodrigues FL, Liberali R, Coutinho VF. Estado nutricional e consumo de alimentos light e diet entre adolescentes da escola privada do município de João Pessoa/PB. Ensaios e Ciências 2010; 14(1):65-68.

16. Maria SHC, Dana CC, Branco LM, Almeida EC, Passos MAZ, Souza E, et al. Consumo alimentar de adolescentes com sobrepeso e obesidade, estudantes de escolas pública e privada do Município de Cotia/SP. Pedriatr. Moderna 2006; 42(2):69-77.

17. Instituto Brasileiro de Geografia e Estatística. Pesquisa de orçamentos familiares 2008/2009. Análise do consumo alimentar pessoal no Brasil. Rio de Janeiro: IBGE; 2010.

18. Piernas C, NG SW, Popkin B. Trends in purchases and intake of foods and beverages containing caloric and low-calorie sweeteners over the last decade in the United States. Pediatric Obesity 2013; 8(4):294-306.

19. Nunes ST, Galon CW. Conhecimento e consumo dos produtos diet e light e a compreensão dos rótulos alimentares por consumidores de um supermercado do município de Caxias do Sul, RS/ Brasil. Nutrire 2013; 38(2):156-171.

20. Sylvetsky AC, Welsh JA, Brown RJ, Vos MB. Low-calorie sweetener consumption is increasing in the United States. Am. J. Clin. Nutr. 2012; 96:640-646.

21. Branco L M, Hilário MOE, Cintra IP. Relação entre o consumo de alimentos dietéticos e light com a condição nutricional, a percepção e a satisfação da imagem corporal em adolescentes. Nutrire 2006; 31(3):27-36

22. Oliveira MBC, Enes CC, Souza CR, Desani DDR, Muniz RP, Salay E. Nível de informação do Consumidor sobre os produtos diet e light em supermercados de Campinas. Rev. Ciênc. Méd. 2005; 14(5):433-440. 
23. Malik VS, Schulze MB, Hu FB. Intake of sugar-sweetened beverages and weight gain: a systematic review. Am. J. Clin. Nutr. 2006; 84:274-88.

24. Ebbeling CB, Feldman HA, Chomitz VR, Antonelli TA, Gortmaker SL, Stavorula KO, et al. A randomized trial of sugar-sweetened beverages and adolescent body weight. N. Engl. J. Med. 2012; 367:1407-1416.

25. Nogueira FAM, Sichieri R. Associação entre consumo de refrigerantes, sucos e leite, com o índice de massa corporal em escolares da rede pública de Niterói, Rio de Janeiro, Brasil. Cad. Saúde Pública 2009; 25(12):2715-2724.

26. Brown RJ, Banate MA, Rother KI. Artificial sweeteners: a systematic review of metabolic effects in youth. Int. J. Pediatr. Obesity 2010; 5(4):305-312.

27. Storey KE, Forbes LE, Fraser SN, Spence JC, Plotnikoff RC, Raine KD, et al. Diet quality, nutrition and physical activity among adolescents: the Web-SPAN (Web-Survey of Physical Activity and Nutrition) project. Public Health Nutrition 2009; 12(11):2009-2017.

28. Bellisle F, Drewnowski A. Intense sweeteners, energy intake and the control of body weight. Eur. J. Clinical Nutrition 2007; 61:691-700.

29. Miller PE, Perez V. Low-calorie sweeteners and body weight and composition: a meta-analysis of randomized controlled trials and prospective cohort studies. Am. J. Clinical Nutrition 2014; 100(3):765-777.

30. Yang Q. Gain weight by 'going diet'? Artificial sweeteners and the neurobiology of sugar cravings. Yale J. Biol. Med. 2010; 83(2):101-108. 